\title{
Commentary: Sensitivity of Fite-faraco Versus Auramine-rhodamine in Mycobacterial Infection
}

\author{
Kelly Atherton BS ${ }^{1 *}$, Latiffa Smith BS ${ }^{2}$, Alan Snyder MD MSCR ${ }^{3}$, John Plante MD MSCR ${ }^{3}$, Dirk Elston MD ${ }^{3}$ \\ 'College of Medicine, Medical University of South Carolina, Charleston, South Carolina; College of Graduate Studies, Medical University of South Carolina, \\ Charleston, South Carolina \\ ${ }^{2}$ College of Medicine, Medical University of South Carolina, Charleston, South Carolina \\ ${ }^{3}$ Department of Dermatology and Dermatologic Surgery, Medical University South Carolina, Charleston, South Carolina
}

\section{Article Info}

\section{Article Notes}

Received: September 06, 2021

Accepted: October 08, 2021

\section{${ }^{*}$ Correspondence:}

${ }^{*}$ Dr. Kelly Atherton, College of Medicine, Medical University of South Carolina, Charleston, South Carolina, USA; Tel: 864-804-9406; Email: atherton@musc.edu.

${ }^{\circledR} 2021$ Atherton $\mathrm{K}$. This article is distributed under the terms of the Creative Commons Attribution 4.0 International License.

\section{Keywords:}

Auramine-rhodamine

Fite-faraco

Mycobacterium

Kinyoun

Ziehl-neelsen

Acid-fast bacilli

\section{Body of Manuscript}

Infection with Nontuberculous Mycobacteria (NTM) species is increasing in prevalence in both endemic and non-endemic areas. ${ }^{1}$ Clinically, NTM infections most commonly present as skin and soft tissue infection, pulmonary disease, lymphadenitis or disseminated disease ${ }^{2}$ but may less likely present in locations such as the eyes, musculoskeletal system or central nervous system (CNS). ${ }^{3}$ When the skin and soft tissues are involved, infection may appear as papules, pustules, plaques, nodules, abscesses, panniculitis, folliculitis, or lymphangitis. Although frequently initially indolent, lesions may ulcerate, and may exhibit proximal lymphangitic spread. ${ }^{4,5}$ Such varied presentations elicit a broad differential of chronic infectious and non-infectious diagnoses. ${ }^{5}$ Chronic, untreated sequelae of cutaneous NTM may be as mild as pigmentary changes or as severe as nerve palsies, permanent disfigurement and bacteremia, depending on the integrity of the host's immune system. ${ }^{6,7}$ Accurate diagnosis of NTM is important to ensure appropriate antibiotic selection.

Although not considered gold standard, histopathologic staining is a common complementary method employed for diagnosis of NTM given that culture can take weeks to result, molecular techniques are not always available in resource-poor areas, and have variable sensitivity. A recently published article, "Sensitivity of Fite-Faraco versus auramine-rhodamine in mycobacterial infection," addressed the efficacy of these two commonly used stains in the histopathological diagnosis of Mycobacteria spp. including NTM and found that of the 53 samples stained positive by auraminerhodamine $(\mathrm{AR}+)$, only $52.8 \%(\mathrm{n}=28)$ were identified as positive by Fite-Faraco (FF). The effectiveness of FF staining was subclassified by biopsy location, resulting in a sensitivity level of 57.1 percent and 68.8 percent in cutaneous and lung tissues, respectively. FF also demonstrated moderate sensitivity in gastrointestinal and lymph node tissues with values of less than 50 percent. $^{8}$

Other notable findings included that, of $17 \mathrm{AR}+$ samples for which polymerase chain reaction (PCR) was also performed, only seven $(41.2 \%)$ were positive by this method. In contrast, of $19 \mathrm{AR}+$ samples for which gene probe was performed, 18 (94.7\%) were positive. Of the 53 total AR+ samples included in the study, 8 (42.1\%) were never identified by species, and $17 \%$ of diagnoses relied on histology alone. ${ }^{8}$ Therefore, there is clearly a need to develop 
diagnostic algorithms for NTM histopathology stains to aid in accuracy and efficiency of diagnosis.

Herein we discuss the strengths, weaknesses, and current uses of the four most common acid-fast histologic stains used for the detection of Mycobacteria, with specific emphasis on NTM. Although this commentary focuses on staining for NTM, the existing literature reviewing the use of these stains for non-tuberculous and non-leprous Mycobacteria species is currently lacking. ${ }^{9}$ Because the same four stains are frequently used for identification of $M$. tuberculosis and M. leprae, the authors have included some references which focus on these species, where existing literature for the application of these stains in NTM is absent.

\section{Auramine-Rhodamine}

Auramine-Rhodamine (AR) is a combination of the fluorescent stains Auramine $\mathrm{O}$ (AO) and Rhodamine $B$ (RB). The method consists of the addition of the AR solution to a slide that has been deparaffinized and placed in distilled water. Following the addition of $\mathrm{AR}$, the section is microwaved for 45 seconds and rinsed with tap water. Potassium permanganate is then added, and the section is rinsed once more and dehydrated; acid-fast organisms typically stain red-yellow, ${ }^{10,11}$ as seen in Fig. 1 . AO, an analogous stain lacking the rhodamine counterpart of $A R$, has been cited to have a sensitivity range of $46-85 \%$ when diagnosing NTM, using FNA and excisional biopsy of lymph tissue respectively. ${ }^{9}$ One study evaluating the performance of $\mathrm{AO}$ and $\mathrm{ZN}$ in 22 patients with clinical histories consistent with NTM lymphadenitis found that AO staining was technically easier and had a higher sensitivity and negative predictive value than $\mathrm{ZN} .{ }^{12} \mathrm{AR}$ has been shown to more sensitive than ZN and FF stains in the detection of M. leprae, with $100 \%$ sensitivity in borderline and indeterminate tuberculoid leprosies in one study. ${ }^{13}$ When used to evaluate M. leprae in 23 skin biopsies that were FF negative but PCR positive, AR fluorescent fragments were identified in 7

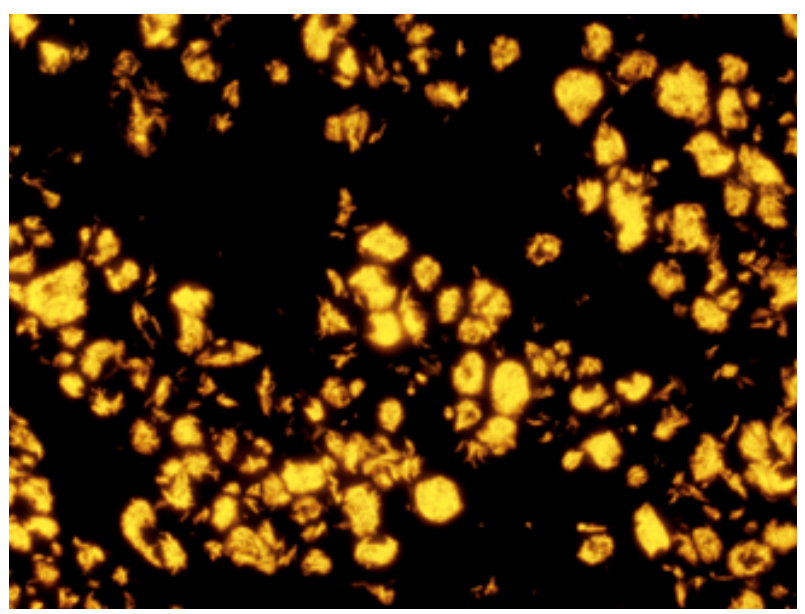

Figure 1: Auramine-Rhodamine stain out of 18 patients. ${ }^{14}$ In another study evaluating M. leprae diagnosis using slit skin smears and punch biopsies, the sensitivity of AO was found to be equal to FF, but superior ease of detection was noted given the strong fluorescence of AO stained organisms against the black background. ${ }^{15}$ Additionally, two studies comparing AR and ZN for the diagnosis of $M$. tuberculosis using saliva/sputum samples and tissue block biopsies, respectively, found that AR was superior to $\mathrm{ZN}{ }^{16,17}$ In a separate manuscript, AR was shown to be equal to or better than Kinyoun staining (KN) for the detection of Cryptosporidium oocytes (acid-fast), though the structure of oocyte cell wall differs substantially from NTM. More importantly, in this study, samples stained with AR required only 2.5 minutes to read on average, whereas KN required a significantly longer 6 minutes, ${ }^{18}$ a function of the technique needed to examine each stain under the microscope. Given its wide availability in the US, relative ease of staining technique, and brief time required for reading of a sample, $\mathrm{AR}$ is the most promising of the stains discussed here for the evaluation of NTM.

\section{Fite-Faraco}

Fite-Faraco (FF) is an inexpensive and commonly used stain in the detection of $M$. leprae in histopathological sections. FF, ZN and KN stains, to be discussed below, are all viewed under light microscopy and classified as carbol-fuchsin stains, a method designed to penetrate the mycolic acid-rich cell walls of mycobacterial organisms. ${ }^{19}$ The staining process of FF consists of deparaffinizing each section with a xylene-peanut oil mixture. Each section is then stained into a carbol fuchsin solution for at least twenty minutes prior to methylene blue counter staining; ${ }^{20}$ typical appearance can be seen in Fig. 2. Previous literature describing the use and effectiveness of FF in NTM diagnosis is largely lacking, a gap which is partially filled by Snyder et al. in the article being commented upon. ${ }^{8}$ Its use has been documented to visualize M. ulcerans in skin samples. ${ }^{21}$ One study that evaluated FF, ZN and PCR for skin punch biopsies of $M$. leprae concluded that FF had a greater sensitivity than $\mathrm{ZN}$, and had the best sensitivity when combined with

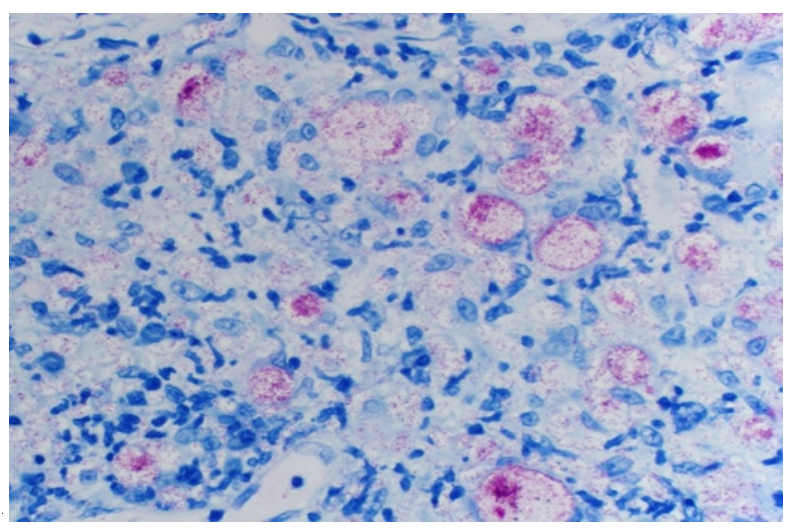

Figure 2: Fite-Faraco stain 
PCR. ${ }^{22}$ In another study examining biopsies of the palatine tonsils and adenoids in suspected M. leprae, FF performed rather poorly, but was tested only on a small portion of the total specimen blocks. ${ }^{23}$ However, FF remains a cheap and valuable method in detecting AFB when other methods cannot be executed.

\section{Ziehl-Neelsen}

Ziehl-Neelsen (ZN), often referred to as modified acid fast stain, is commonly used in detecting pulmonary tuberculosis, and has been variably documented for detection of NTM. The staining procedure consists of deparaffinizing sections through xylene and rinsing with absolute alcohol and distilled water. Sections are then stained with carbol fuchsin solution for at least ten minutes and heat applied, then subsequently rinsed in water. After adding carbol fuchsin, sections are decolorized with acid alcohol until the hue appears pale-pink, as seen in Fig. 3. Following decolorization, sections are washed with water, and counter stained with methylene blue. To complete the process, slides are dehydrated with alcohol and cleared with xylene..$^{20,24}$ It should be noted that safety concerns exist for laboratory workers in the form of exposure to phenol, which is produced during the heating process. ${ }^{25}$ In one retrospective study that reviewed FNA specimens in confirmed $M$. fortuitum cervical lymphadenitis in patients with AIDS, AO, KN and ZN performed relatively similarly, with sensitivities of $36 \%, 43 \%$ and $33 \%$ respectively. ${ }^{26}$ In one report, $\mathrm{ZN}$ was successfully used to identify $M$. fortuitum from a vegetation in the setting of infective endocarditis. ${ }^{27}$ This method is historically less effective in staining leprosy and other NTM, compared to tuberculosis, due to its inefficient absorption profiles for acid and alcohol, but it can be used as a general method to decrease background staining of sputum with vigorous decolorization. ${ }^{26}$

\section{Kinyoun}

Kinyoun (KN) is commonly used for the detection of acid-fast bacilli. Whereas the ZN staining technique involves heating the sample in order to enhance stain penetration of

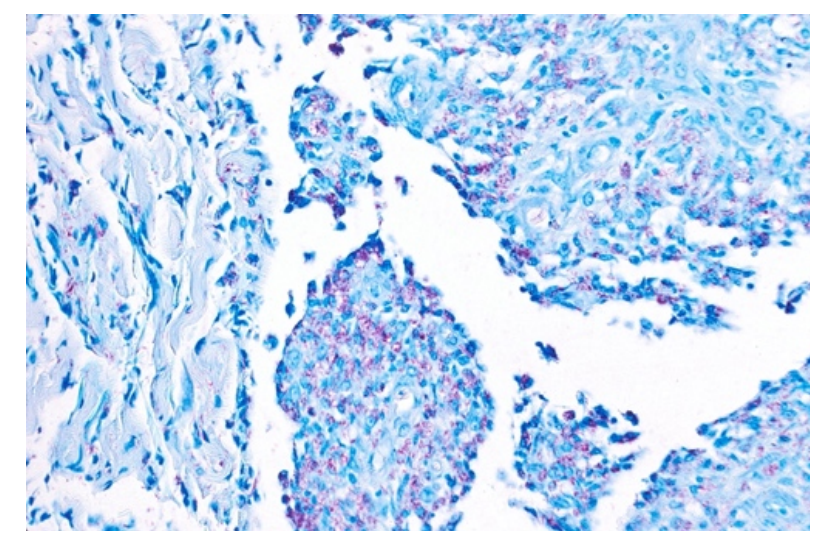

Figure 3: Ziehl-Neelsen stain the cell wall, KN is a "cold staining" method, which relies on a higher concentration of carbol-fuchsin stain, rather than heat, to adequately penetrate the cell wall. ${ }^{28} \mathrm{KN}$ staining is performed by applying the carbol-fuchsin solution for one hour at room temperature, applying 1\% acid alcohol until the tissue sample is pale pink, and counterstaining with a methylene blue solution, resulting in a bright red appearance of the acid-fast organisms, with a light blue background, ${ }^{20}$ as seen in Fig. 4 . While KN staining is relatively easy from a technical perspective, one major drawback of the modified KN technique is that it requires 24 hours for completion, limiting the possibility of rapid diagnosis. ${ }^{29} \mathrm{KN}$ has been used to detect a variety of acid-fast organisms $\mathrm{s}^{30}$ but, to date, is only variably used or reported in the detection of NTM. ${ }^{31,32}$ However, its use and accuracy have been well reported in its application to tuberculosis diagnostics, with $\mathrm{KN}$ documented to be inferior to $\mathrm{ZN}^{33,32}$ and $\mathrm{AO}^{34}$ for the purpose of detecting tuberculosis in sputum samples, while another study found that the modified KN stain was comparable to ZN, with a $99.5 \%$ agreement rate. ${ }^{25}$ Yet, these studies have not frequently relied on methods of direct comparison between stains, emphasizing the need for future research into the efficacy of KN, especially as applied to NTM.

In summary, from the available literature, $A R$ is the preferred stain of choice for mycobacterial infection diagnosis via histopathology, but FF may be a reasonable alternative in resource-poor environments without access to fluorescence microscopy. ${ }^{35,36}$ There is not enough information regarding the use of AR or KN in NTM specifically to make recommendations regarding their use. The staining methods discussed above do have other limitations beyond their ability to detect NTM. A summary of the available sensitivities of each stain is provided in Table 1. NTM histology is not species-specific and is typically characterized by a suppurative, granulomatous reaction and necrosis with stellate abscesses and draining sinus tracts. ${ }^{15}$ Of note, NTM histology in immunocompromised individuals may be cryptic secondary to improper cutaneous immune

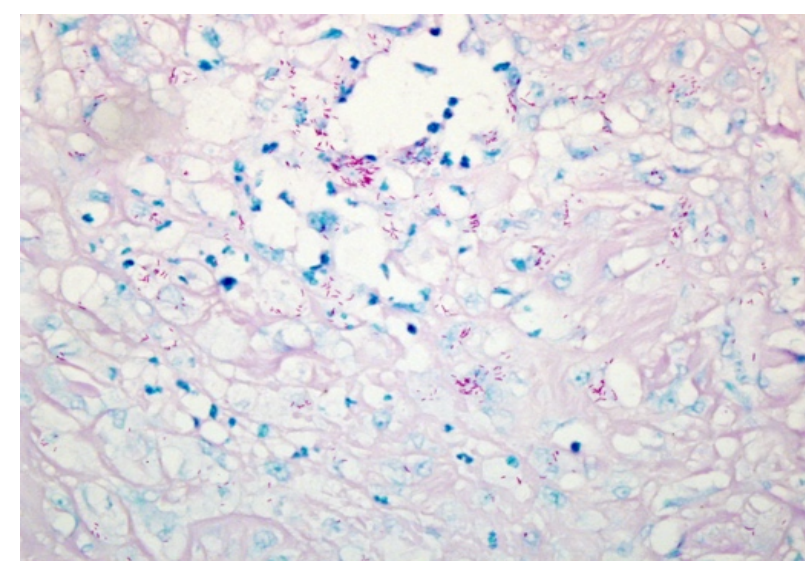

Figure 4: Kinyoun stain 
Table 1: Overall attributes and known sensitivities of AR, FF, ZN and KN for non-leprous NTM detection in histopathology smears, organized by tissue type

\begin{tabular}{|l|c|c|c|c|c|c|}
\hline \multicolumn{1}{|c|}{} & \multicolumn{4}{c|}{ Tissue Type } \\
\hline Auramine-Rhodamine & Overall Attributes & Skin & Lung & Sputa & GI & FNA/Lymph \\
\hline Fite-Faraco & Fluorescent; most ease of viewing & $*$ & $*$ & $*$ & $*$ & $36 \%^{26}-85 \%^{9}$ \\
\hline Ziehl-Neelsen & Carbol-Fuchsin & $57.1 \%^{8}$ & $58.8 \%^{8}$ & $*$ & $<50 \%^{8}$ & $<50 \%^{8}-$ \\
\hline Kinyoun & $\begin{array}{c}\text { Carbol-Fuchsin; Requires heat; Safety } \\
\text { concerns }\end{array}$ & $*$ & $*$ & $*$ & $*$ & $33 \%^{25}$ \\
\hline
\end{tabular}

*No existing literature documenting sensitivity of stain in tissue type

response, and yet, pre-stain microorganism viability has been found to be irrelevant for staining quality in $A R, Z N$, and $\mathrm{KN}^{37}$

Beyond the histopathology methods discussed above, culture and PCR remain as gold standard for diagnosis of NTM infections. However, culture can require weeks to allow for colony growth, and in Snyder et al., culture was found to be positive only $75 \%$ of the time in cases of NTM infection proven via other methods. ${ }^{8}$ Therefore, various reflexive molecular techniques may be used in conjunction with culture for rapid species detection and subsequent identification, ${ }^{38}$ with methods including PCR, gene probe, DNA sequencing, high-performance liquid chromatography (HPLC), and MALDI-TOF mass spectrometry. PCR uses a short, single-stranded DNA primer that allows for amplification of a specific DNA sequence, through the process of denaturing, annealing and extending. The resulting amplified sequence can then be further analyzed for species-specific polymorphisms that can be detected by additional methods, such as gel electrophoresis. Similarly, gene probe is a targeted nucleic acid hybridization tool that uses single stranded DNA or RNA for complementary binding in order to target specific genes for amplification. DNA sequencing, whether whole-exome or whole-genome, involves the determination of the nucleic acid sequence of a species' DNA, which allows for comparison and speciation of subsequent samples. HPLC utilizes molecular analysis of proteins through the passage of a pressurized sample through an absorbent column, resulting in identification, via a detector, of unique molecular signatures specific to bacterium. MALDI-TOF utilizes extracted ribosomal proteins which are ionized, becoming electrically charged and vaporized, and separated by their mass-to-charge ratio, allowing for identification of molecular components. ${ }^{39}$ Each of these diagnostic technologies can be employed to detect and speciate NTM in various settings.

Overall, literature regarding the efficacy of the four stains discussed above is lacking, specifically concerning their use for NTM detection. This should constitute an area of further research, as histopathologic staining remains one of several diagnostic tools for clinically elusive NTM infections, despite the more recent development of numerous molecular tools. Perhaps the most convincing argument for further stain characterization lies in the fact that once histopathologic diagnosis of NTM is made, treatment can begin sooner, while awaiting concomitant molecular testing results for speciation purposes. Once NTM is speciated, treatment can be adjusted as appropriate. Overall, literature regarding the efficacy of the four stains discussed above is lacking, specifically concerning their use for NTM detection. This should constitute an area of further research, as histopathologic staining remains one of several diagnostic tools for clinically elusive NTM infections, despite the more recent development of numerous molecular tools. Perhaps the most convincing argument for further stain characterization lies in the fact that once histopathologic diagnosis of NTM is made, treatment can begin sooner, while awaiting concomitant molecular testing results for speciation purposes. Once NTM is speciated, treatment can be adjusted as appropriate.

\section{Acknowledgments}

\section{None}

\section{Conflicts of Interest}

All authors declare no conflicts of interest.

\section{Funding}

None

\section{References}

1. Wentworth $A B$, Drage LA, Wengenack NL, et al. Increased incidence of cutaneous nontuberculous mycobacterial infection, 1980 to 2009: a population-based study. Mayo Clin Proc. 2013 Jan; 88(1): 38-45. doi: 10.1016/j.mayocp.2012.06.029. Epub 2012 Dec 4. PMID: 23218797; PMCID: PMC3690780.

2. Diagnosis and treatment of disease caused by nontuberculous mycobacteria. This official statement of the American Thoracic Society was approved by the Board of Directors, March 1997. Medical Section of the American Lung Association. Am J Respir Crit Care Med. 1997 Aug; 156(2 Pt 2): S1-25. doi: 10.1164/ajrccm.156.2.atsstatement. PMID: 9279284.

3. Cai R, Qi T, Lu H. Central nervous system infection with nontuberculous mycobacteria: a report of that infection in two patients with AIDS. Drug Discov Ther. 2014 Dec; 8(6): 276-9. doi: 10.5582/ ddt.2014.01047. PMID: 25639308.

4. Misch EA, Saddler C, Davis JM. Skin and Soft Tissue Infections Due to Nontuberculous Mycobacteria. Curr Infect Dis Rep. 2018 Mar 19; 20(4): 6. doi: 10.1007/s11908-018-0611-3. PMID: 29556857. 
5. Elston D. Nontuberculous mycobacterial skin infections: recognition and management. Am J Clin Dermatol. 2009; 10(5): 281-5. doi: 10.2165/00128071-200910050-00001. PMID: 19658440.

6. Mahadevan M, Neeff M, Van Der Meer G, et al. Non-tuberculous mycobacterial head and neck infections in children: Analysis of results and complications for various treatment modalities. Int J Pediatr Otorhinolaryngol. 2016 Mar; 82: 102-6. doi: 10.1016/j. ijporl.2015.12.026. Epub 2016 Jan 7. PMID: 26857325.

7. Lim JM, Kim JH, Yang HJ. Management of infections with rapidly growing mycobacteria after unexpected complications of skin and subcutaneous surgical procedures. Arch Plast Surg. 2012 Jan; 39(1): 18-24. doi: 10.5999/aps.2012.39.1.18. Epub 2012 Jan 15. PMID: 22783486; PMCID: PMC3385308.

8. Snyder AN, O'Connor H, Plante JG, et al. Sensitivity of Fite-Faraco versus auramine-rhodamine in mycobacterial infection. J Am Acad Dermatol. 2020 Jul 8: S0190-9622(20)32166-6. doi: 10.1016/j. jaad.2020.07.002. Epub ahead of print. PMID: 32652190.

9. Willemse SH, Oomens MAEM, De Lange J, et al. Diagnosing nontuberculous mycobacterial cervicofacial lymphadenitis in children: A systematic review. Int J Pediatr Otorhinolaryngol. 2018 Sep; 112: 48-54. doi: 10.1016/j.ijporl.2018.06.034. Epub 2018 Jun 19. PMID: 30055739.

10. Carson F, Histotechnology: A Self-Instructional Text, 1990, pp192193, ASCP, ILL

11. Crookham,J, Dapson,R, Hazardous Chemicals in the Histopathology Laboratory, 2nd ED, 1991, Anatech

12. Kommareddi S, Abramowsky CR, Swinehart GL, et al. Nontuberculous mycobacterial infections: comparison of the fluorescent auramine- 0 and Ziehl-Neelsen techniques in tissue diagnosis. Hum Pathol. 1984 Nov;15(11):1085-9. doi: 10.1016/s0046-8177(84)80253-1. PMID: 6208117.

13. Adiga DS, Hippargi SB, Rao G, et al. Evaluation of Fluorescent Staining for Diagnosis of Leprosy and its Impact on Grading of the Disease: Comparison with Conventional Staining. J Clin Diagn Res. 2016 Oct; 10(10): EC23-EC26. doi: 10.7860/JCDR/2016/22470.8739. Epub 2016 Oct 1. PMID: 27891346; PMCID: PMC5121684.

14. Elston DM, Liranzo MO, Scollard DM. Comparing the sensitivity of auramine-rhodamine fluorescence to polymerase chain reaction in the detection of Mycobacterium leprae in Fite-negative tissue sections. J Am Acad Dermatol. 2017 May; 76(5): 992-993. doi: 10.1016/j.jaad.2016.11.045. PMID: 28411775.

15. Girma S, Avanzi C, Bobosha K, et al. Evaluation of Auramine 0 staining and conventional PCR for leprosy diagnosis: A comparative crosssectional study from Ethiopia. PLoS Negl Trop Dis. 2018 Sep 4; 12(9): e0006706. doi: 10.1371/journal.pntd.0006706. PMID: 30180155; PMCID: PMC6138420.

16. Holani AG, Ganvir SM, Shah NN, et al. Demonstration of mycobacterium tuberculosis in sputum and saliva smears of tuberculosis patients using ziehl neelsen and flurochrome staining- a comparative study. J Clin Diagn Res. 2014 Jul; 8(7): ZC42-5. doi: 10.7860/ JCDR/2014/9764.4587. Epub 2014 Jul 20. PMID: 25177636; PMCID: PMC4149142.

17. Ahmad F, Rehman A, Jadoon O. Histological Comparison of Two Staining Methods In Identification Of Mycobacterium Tuberculosis In Granulomatous Lymphadenitis. J Ayub Med Coll Abbottabad. 2018 Apr-Jun; 30(2): 159-162. PMID: 29938410.

18. Tortora GT, Malowitz R, Mendelsohn B, et al. Rhodamine-auramine 0 versus Kinyoun-carbolfuchsin acid-fast stains for detection of Cryptosporidium oocysts. Clin Lab Sci. 1992 Nov-Dec; 5(6): 568-9. PMID: 10150976.

19. Madison BM. Application of stains in clinical microbiology. Biotech Histochem. 2001 May; 76(3): 119-25. PMID: 11475314
20. Gridley MF, Ambrooi LP, editors. Manual of Histologic and Special Staining Techniques. Ed. 2. The Armed Forces Institute of Pathology, Washington, D.C.; 1957. 206 p.

21. Mwanatambwe M, Fukunishi Y, Yajima M, et al. Clinicohistopathological findings of Buruli ulcer. Nihon Hansenbyo Gakkai Zasshi. 2000 Jul;69(2):93-100. doi: 10.5025/hansen.69.93. PMID: 10979276.

22. Reja AH, Biswas N, Biswas S, et al. Fite-Faraco staining in combination with multiplex polymerase chain reaction: a new approach to leprosy diagnosis. Indian J Dermatol Venereol Leprol. 2013 Sep-Oct; 79(5): 693-700. doi: 10.4103/0378-6323.116740. PMID: 23974586

23. Morgado de Abreu MAM, Nai GA, Molina JD, et al. Mycobacterium leprae on Palatine Tonsils and Adenoids of Asymptomatic Patients, Brazil. Emerg Infect Dis. 2020 Oct; 26(10): 2518-2520. doi: 10.3201/ eid2610.191267. PMID: 32946726; PMCID: PMC7510695.

24. Bancroft JD, Stevens A. Theory and practice of histological techniques. Fourth edition. New York: Churchill Livingstone; 1996.

25. Kurup R, Chester K. Comparative evaluation of ziehl neelsen staining and knowledge, attitudes and practices of laboratory personnel in relation to ziehl nielsen. West Indian Med J. 2014; 63(1): 34-39. doi:10.7727/wimj.2012.247

26. Smith MB, Schnadig VJ, Boyars MC, et al. Clinical and pathologic features of Mycobacterium fortuitum infections. An emerging pathogen in patients with AIDS. Am J Clin Pathol. 2001 Aug; 116(2): 225-32. doi: 10.1309/HF2V-E8WV-PX4Q-CHQH. PMID: 11488069 .

27. Sharma H, Keshavan A, Little MA, et al. Fortuitous vasculitis. Ren Fail. 2012; 34(3): 378-82. doi: 10.3109/0886022X.2011.647337. Epub 2012 Jan 17. PMID: 22250755.

28. Singhal R, Myneedu VP. Microscopy as a diagnostic tool in pulmonary tuberculosis. Int J Mycobacteriol. 2015 Mar; 4(1): 1-6. doi: 10.1016/j. ijmyco.2014.12.006. Epub 2015 Feb 2. PMID: 26655191.

29. Zhao D, Yang XM, Chen QY, et al. A modified acid-fast staining method for rapid detection of Mycobacterium tuberculosis. J Microbiol Methods. 2012 Oct;91(1):128-32. doi: 10.1016/j.mimet.2012.07.024. Epub 2012 Aug 11. PMID: 22902528.

30. Salleh FM, Al-Mekhlafi AM, Nordin A, et al. Evaluation of gramchromotrope kinyoun staining technique: its effectiveness in detecting microsporidial spores in fecal specimens. Diagn Microbiol Infect Dis. 2011 Jan; 69(1): 82-5. doi: 10.1016/j.diagmicrobio.2010.08.028. PMID: 21146718.

31. Philips RC, Hoyer PE, White SM, et al. Cutaneous nontuberculous mycobacteria infections: A retrospective case series of 78 patients from the Texas Gulf Coast region. J Am Acad Dermatol. 2019 Sep; 81(3): 730-739. doi: 10.1016/j.jaad.2019.04.022. Epub 2019 Apr 16. PMID: 31002850.

32. Wu RI, Mark EJ, Hunt JL. Staining for acid-fast bacilli in surgical pathology: practice patterns and variations. Hum Pathol. 2012 Nov; 43(11): 1845-51. doi: 10.1016/j.humpath.2012.01.006. Epub 2012 Apr 26. PMID: 22542129.

33. Sawadogo TL, Savadogo LG, Diande S, et al. Comparaison des performances des colorations de Kinyoun et à l'auramine 0 , avec celle de Ziehl-Neelsen pour le diagnostic de la tuberculose au Centre national de lutte antituberculeuse du Burkina Faso [Comparison of Kinyoun, auramine 0 , and Ziehl-Neelsen staining for diagnosing tuberculosis at the National Tuberculosis Center in Burkina Faso]. Med Sante Trop. 2012 Jul-Sep; 22(3): 302-6. French. doi: 10.1684/ mst.2012.0082. PMID: 23174139.

34. Van Deun A, Hamid Salim A, Aung KJ, et al. Performance of variations of carbolfuchsin staining of sputum smears for AFB under field conditions. Int J Tuberc Lung Dis. 2005 Oct; 9(10): 1127-33. PMID: 16229224. 
35. Forbes BA, Hall GS, Miller MB, et al. Practice Guidelines for Clinical Microbiology Laboratories: Mycobacteria. Clin Microbiol Rev. 2018 Jan 31; 31(2): e00038-17. doi: 10.1128/CMR.00038-17. PMID: 29386234; PMCID: PMC5967691.

36. Pfyffer GE. 2015. Mycobacterium: general characteristics, laboratory detection, and staining procedures, p 536-569. In Jorgensen JH, Pfaller MA, Carroll KC, Funke G, Landry ML, Richter SS, Warnock DW (ed), Manual of clinical microbiology, 11th ed, vol 1. ASM Press, Washington, DC.

37. Truant AL, Conaron J, Moghaddas J. Effect of pre-stain viability on the acid-fast staining characteristics of Mycobacterium species. Diagnostic Microbiology and Infectious Disease. 2001; 39(2): 121-123
38. De Beenhouwer H, Liang Z, De Rijk P, et al. Detection and identification of mycobacteria by DNA amplification and oligonucleotide-specific capture plate hybridization. J Clin Microbiol. 1995 Nov; 33(11): 29948. doi: 10.1128/JCM.33.11.2994-2998.1995. PMID: 8576360; PMCID: PMC228621.

39. Alcaide F, Amlerová J, Bou G, et al. European Study Group on Genomics and Molecular Diagnosis (ESGMD). How to: identify non-tuberculous Mycobacterium species using MALDI-TOF mass spectrometry. Clin Microbiol Infect. 2018 Jun; 24(6): 599 603. doi: 10.1016/j.cmi.2017.11.012. Epub 2017 Nov 22. PMID: 29174730 